\title{
Additive representation in short intervals, I: Waring's problem for cubes
}

\author{
J. Brüdern and T. D. Wooley
}

\begin{abstract}
Estimates are established for the number of integers of size $N$, in intervals of size $N^{\theta}$, that fail to admit a representation as the sum of $s$ cubes $(s=5,6)$. Thereby it is shown that almost all such integers are represented in the proposed manner. When $s=5$ one may take $\theta=10 / 21$, and when $s=6$ one may take any $\theta>17 / 63$. Similar such conclusions are also established for the related problem associated with the expected asymptotic formula.
\end{abstract}

\section{Introduction}

Although available technology frequently fails to establish that all large integers are represented in some prescribed additive form, there are many situations in which one is nonetheless able to conclude that almost all such integers are thus represented. When the numbers that we seek to represent lie in some thin subsequence of the integers, the existence of such a conclusion might reasonably be construed as additional evidence in favour of the assertion that all large integers are represented in the given form. Pursuing this line of enquiry, a sequence of papers arising from work of the authors jointly with Kawada has been devoted to the investigation of exceptional sets primarily in thin polynomial sequences [BKW01a, BKW00, BKW01b, BKW03], and also in certain arithmetic sequences such as the set of prime numbers [BKW02]. On this occasion, we turn our attention to the problem of establishing strong exceptional set estimates in short intervals, a topic that has already received much attention in the literature (see especially [BHP97, BW95, Kaw96, LP96, PP93, Ram73]). Although our ideas in this context are quite widely applicable, we concentrate in this paper on applications to Waring's problem for cubes. The reader will experience no difficulty in generalising our results to analogous problems involving higher powers.

Denote by $E_{s}(N)$ the number of natural numbers up to $N$ that cannot be written as the sum of $s$ cubes of natural numbers. After work of Linnik [Lin43] and Davenport [Dav39], respectively, it is known that $E_{s}(N) \ll 1$ for $s \geqslant 7$, and that $E_{s}(N)=o(N)$ for $s \geqslant 4$. Indeed, the most recent developments in the circle method permit one to show that, whenever $\varepsilon$ is a sufficiently small positive number, then

$$
E_{4}(N) \ll N^{37 / 42-\varepsilon}, \quad E_{5}(N) \ll N^{5 / 7-\varepsilon} \quad \text { and } \quad E_{6}(N) \ll N^{23 / 42-\varepsilon}
$$

(see Brüdern [Bru91] and Wooley [Woo95, Woo00] for the first of these estimates, and the discussion in $\S 1$ of Brüdern, Kawada and Wooley [BKW01a] for the second and third estimates). When $4 \leqslant s \leqslant 6$, denote by $\beta_{s}$ the least non-negative number satisfying the property that, whenever

Received 7 December 2001, accepted in final form 7 May 2002.

2000 Mathematics Subject Classification 11P05, 11P55.

Keywords: Waring's problem, exceptional sets, short intervals.

The second author is supported in part by NSF grant DMS-9970440. This paper was largely written while the second author was enjoying the generous hospitality and support of Harvard University.

This journal is (C) Foundation Compositio Mathematica 2004. 


\section{J. BRÜDERN AND T. D. WOOLEY}

$\theta>\beta_{s}$, and $\eta$ is a sufficiently small positive number, then one has

$$
E_{s}\left(N+N^{\theta}\right)-E_{s}(N) \ll N^{\theta-\eta} .
$$

In view of (1.1), it is of course trivial that $\beta_{4}<37 / 42, \beta_{5}<5 / 7$ and $\beta_{6}<23 / 42$.

The first non-trivial results concerning exceptional sets for sums of cubes in short intervals are due to Brüdern and Watt, who established that $\beta_{4} \leqslant 3 / 4$ (see Theorem 2 of [BW95]). This conclusion was subsequently improved by Kawada [Kaw96], who proved that

$$
\beta_{4} \leqslant 1585 / 2169=0.730751 \ldots .
$$

Note that whenever almost all integers in the interval

$$
\left[N-\left[N^{1 / 3}\right]^{3}, N-\left[N^{1 / 3}\right]^{3}+M\right]
$$

are sums of $s$ cubes of natural numbers, then almost all integers in the interval $[N, N+M]$ are sums of $s+1$ cubes of natural numbers. It is therefore apparent that $\beta_{s} \leqslant \frac{2}{3} \beta_{s-1}(s=5,6)$, whence, in particular, Kawada's bound (1.2) leads to the bounds

$$
\beta_{5}<0.487168 \text { and } \beta_{6}<0.324779 \text {. }
$$

Following the development of an auxiliary mean value estimate in $\S 2$, we are able to exploit the ideas underlying papers in our earlier series jointly with Kawada in order to establish, in $\S 3$, a conclusion that yields a further improvement in the estimate for $\beta_{6}$ provided in (1.3).

Theorem 1.1. Whenever $17 / 63<\theta \leqslant 1$, and $\eta$ is a sufficiently small positive number, one has

$$
E_{6}\left(N+N^{\theta}\right)-E_{6}(N) \ll N^{\theta-\eta} .
$$

In particular, one has

$$
\beta_{6} \leqslant 17 / 63=0.269841 \ldots
$$

We remark that by incorporating the mean value estimates of Wooley [Woo00] into the arguments of Brüdern [Bru91] underlying Lemma 3.2 of [BKW01a], one would obtain the slightly sharper upper bound $\beta_{6}<0.269674$. We are also able to establish a bound for $\beta_{5}$ slightly sharper than that recorded in (1.3). In this instance our improvements are more modest than those embodied in Theorem 1.1, and the associated methods are considerably more complicated. Following the preparation of an auxiliary estimate in $\S 4$, we therefore defer the proof of this new bound to $\S 5$.

Theorem 1.2. Whenever $10 / 21 \leqslant \theta \leqslant 1$, and $\eta$ is a sufficiently small positive number, one has

$$
E_{5}\left(N+N^{\theta}\right)-E_{5}(N) \ll N^{\theta-\eta} .
$$

In particular, one has

$$
\beta_{5} \leqslant 10 / 21=0.476190 \ldots
$$

We turn our attention next to analogues of the above exceptional set problems in which one seeks to show that the expected asymptotic formula for the number of representations holds almost always. Denote by $R_{s}(n)$ the number of representations of $n$ as the sum of $s$ cubes of natural numbers. A heuristic application of the circle method suggests that, for $s \geqslant 4$, one should have

$$
R_{s}(n)=\frac{\Gamma(4 / 3)^{s}}{\Gamma(s / 3)} \mathfrak{S}_{s}(n) n^{s / 3-1}+o\left(n^{s / 3-1}\right),
$$

where

$$
\mathfrak{S}_{s}(n)=\sum_{q=1}^{\infty} \sum_{\substack{a=1 \\(a, q)=1}}^{q}\left(q^{-1} \sum_{r=1}^{q} e\left(a r^{3} / q\right)\right)^{s} e(-n a / q),
$$




\section{ADDITIVE REPRESENTATION IN SHORT INTERVALS}

and $e(z)$ denotes $e^{2 \pi i z}$. It is known that, for $s \geqslant 4$, the singular series $\mathfrak{S}_{s}(n)$ satisfies the lower bound $\mathfrak{S}_{s}(n) \gg 1$ (see, for example, Theorem 4.5 of Vaughan [Vau97]), and so the relation (1.4) does indeed constitute an asymptotic formula. In order to measure the frequency with which the expected asymptotic formula (1.4) might fail, when $\psi(\tau)$ is a function of a positive variable $\tau$, we define $\widetilde{E}_{s}(N ; \psi)$ to be the number of integers $n$ with $1 \leqslant n \leqslant N$ for which

$$
\left|R_{s}(n)-\frac{\Gamma(4 / 3)^{s}}{\Gamma(s / 3)} \mathfrak{S}_{s}(n) n^{s / 3-1}\right|>n^{s / 3-1} \psi(n)^{-1} .
$$

As was essentially pointed out in [BKW01b], it follows from work of Vaughan [Vau86a], as refined by Boklan [Bok93], that whenever $\psi(\tau)$ is an increasing function growing sufficiently slowly, then one has

$$
\widetilde{E}_{4+t}(N ; \psi) \ll N^{1-t / 6}(\log N)^{\varepsilon-3+t / 2} \psi(N)^{2} \quad(0 \leqslant t \leqslant 3) .
$$

Moreover, the celebrated work of Vaughan [Vau86a] shows, under the same hypotheses on $\psi$, that $\widetilde{E}_{8}(N ; \psi) \ll 1$. The bound (1.7) has recently been sharpened by Wooley [Woo02] in the case $t=3$ to obtain

$$
\widetilde{E}_{7}(N ; \psi) \ll N^{4 / 9+\varepsilon} .
$$

Developing the ideas from our previous work [BKW01b], jointly with Kawada, concerning asymptotic formulae, we establish in $\S 6$ a short intervals analogue of the above bounds for $\widetilde{E}_{s}(N ; \psi)$ for $5 \leqslant s \leqslant 7$.

TheOREM 1.3. Suppose that $\psi(\tau)$ is a function of a positive variable $\tau$, increasing monotonically to infinity, and satisfying $\psi(\tau)=O\left(\tau^{\delta}\right)$ for some sufficiently small positive number $\delta$. Also, let $M$ and $N$ be large positive numbers with $M \leqslant N$. Then for $1 \leqslant t \leqslant 3$, and for each positive number $\varepsilon$, one has

$$
\widetilde{E}_{4+t}(N+M ; \psi)-\widetilde{E}_{4+t}(N ; \psi) \ll\left(N^{(5-t) / 6}+M N^{(1-t) / 6}\right)(\log N)^{\varepsilon-(7-t) / 2} \psi(N)^{2} .
$$

Plainly, the conclusion of Theorem 1.3 provides non-trivial estimates for exceptional sets of integers of size $N$, in short intervals of size

$$
\begin{array}{ll}
N^{2 / 3}(\log N)^{\varepsilon-3}, & \text { for sums of five cubes, } \\
N^{1 / 2}(\log N)^{\varepsilon-5 / 2}, & \text { for sums of six cubes, } \\
N^{1 / 3}(\log N)^{\varepsilon-2}, & \text { for sums of seven cubes. }
\end{array}
$$

For sums of four cubes, meanwhile, one has the earlier conclusion of Brüdern and Watt [BW95] demonstrating that, whenever $M=N^{\theta}$ with $5 / 6<\theta<1$, then one has

$$
\widetilde{E}_{4}\left(N+M ;(\log \tau)^{1 / 5}\right)-\widetilde{E}_{4}\left(N ;(\log \tau)^{1 / 5}\right) \ll M(\log N)^{-1 / 4} .
$$

It is evident that both the latter conclusion, and the estimates stemming from Theorem 1.3, go beyond what is trivially available via the upper bounds (1.7) and (1.8). Perhaps it is worth remarking also that the argument used to establish Theorem 1.3 is easily adapted to show that, whenever $M=N^{\theta}$ with $\theta>1 / 6$, and $\delta$ is a sufficiently small positive number satisfying $2 \delta<\theta-1 / 6$, then one has

$$
\widetilde{E}_{8}\left(N+M ; \tau^{\delta}\right)-\widetilde{E}_{8}\left(N ; \tau^{\delta}\right) \ll M N^{-\delta},
$$

a conclusion that goes beyond that automatically available from the aforementioned result of Vaughan concerning sums of eight cubes.

Since the basic plan of attack in such problems is described in detail within our earlier paper [BKW01a] jointly with Kawada, we avoid discussing details of strategy at this point. It is sufficient to remark that we encode information concerning exceptional integers within an exponential sum, and then exploit this exponential sum explicitly via mean value estimates familiar to those expert 


\section{J. BRÜDERn AND T. D. WOOLEY}

in applications of the circle method. This approach retains the local information concerning the set of exceptions that is more difficult to exploit via more traditional approaches involving the use of Bessel's inequality.

Throughout, the letter $\varepsilon$ will denote a sufficiently small positive number. We take $P$ to be the basic parameter, a large real number depending at most on $\varepsilon$. We use $\ll$ and $\gg$ to denote Vinogradov's well-known notation, implicit constants depending at most on $\varepsilon$. Sometimes we make use of vector notation. For example, the expression $\left(c_{1}, \ldots, c_{t}\right)$ is abbreviated to $\mathbf{c}$. Also we write $[x]$ for the greatest integer not exceeding $x$. In an effort to simplify our analysis, we adopt the following convention concerning the parameter $\varepsilon$. Whenever $\varepsilon$ appears in a statement, we assert that for each $\varepsilon>0$ the statement holds for sufficiently large values of the main parameter. Note that the 'value' of $\varepsilon$ may consequently change from statement to statement, and hence also the dependence of implicit constants on $\varepsilon$.

\section{An auxiliary mean value estimate}

We establish in this section a mean value estimate crucial to the strength of Theorem 1.1. Before announcing this bound, we introduce some notation. Let $P$ be a large positive number, and take $M$ to be a real number with $1 \leqslant M \leqslant P^{1 / 3}$. We then write $Q=P M^{-1}$ and $H=P M^{-3}$. Next let $N$ and $Z$ be large positive numbers, and suppose that $\mathcal{Z}$ is a set of integers with $\mathcal{Z} \subseteq[N, N+Z]$. It is convenient to abbreviate $\operatorname{card}(\mathcal{Z})$ simply to $\widehat{Z}$. Finally, we introduce the exponential sums

$$
f(\alpha)=\sum_{P<x \leqslant 2 P} e\left(\alpha x^{3}\right), \quad g(\alpha)=\sum_{Q<y \leqslant 2 Q} e\left(\alpha y^{3}\right), \quad K(\alpha)=\sum_{n \in \mathcal{Z}} e(n \alpha) .
$$

Lemma 2.1. Suppose that the parameters $M, P, Q, Z$, and the quantity $\widehat{Z}$, satisfy the inequalities

$$
M^{3} \leqslant \widehat{Z} \leqslant Z \leqslant \min \left\{2 P, Q^{2}\right\} .
$$

Then for each positive number $\varepsilon$, one has

$$
\int_{0}^{1}|f(\alpha) g(\alpha) K(\alpha)|^{2} d \alpha \ll P^{\varepsilon}\left(\left(P+P^{1 / 2} H\right) Q \widehat{Z}+(P Q)^{1 / 2}\left(P+H^{2}\right)^{1 / 2} \widehat{Z}^{3 / 2}\right) .
$$

Proof. On considering the underlying diophantine equation, it follows from orthogonality that the integral on the left-hand side of (2.1) is equal to the number of solutions of the diophantine equation

$$
x_{1}^{3}-x_{2}^{3}=y_{1}^{3}-y_{2}^{3}+n_{1}-n_{2},
$$

with $P<x_{i} \leqslant 2 P(i=1,2), Q<y_{j} \leqslant 2 Q(j=1,2)$ and $n_{l} \in \mathcal{Z}(l=1,2)$. Given any solution $\mathbf{x}$, $\mathbf{y}, \mathbf{n}$ counted by the latter equation, it is evident from our hypotheses on $Z$ that

$$
\left|y_{1}^{3}-y_{2}^{3}+n_{1}-n_{2}\right|<(2 Q)^{3}+Z<9 Q^{3} .
$$

Meanwhile, whenever $x_{1} \neq x_{2}$, one has

$$
9 Q^{3}>\left|x_{1}^{3}-x_{2}^{3}\right|>3 P^{2}\left|x_{1}-x_{2}\right|,
$$

and thus $\left|x_{1}-x_{2}\right|<3 H$. In the latter situation, on substituting $z=x_{1}+x_{2}$ and $h=x_{1}-x_{2}$, we deduce from (2.2) that

$$
h\left(3 z^{2}+h^{2}\right)=4\left(y_{1}^{3}-y_{2}^{3}+n_{1}-n_{2}\right),
$$

wherein $1 \leqslant z \leqslant 4 P$ and $1 \leqslant|h| \leqslant 3 H$. Write

$$
F(\alpha)=\sum_{1 \leqslant h \leqslant 3 H} \sum_{1 \leqslant z \leqslant 4 P} e\left(\alpha h\left(3 z^{2}+h^{2}\right)\right) .
$$




\section{ADDITIVE REPRESENTATION IN SHORT INTERVALS}

Then on considering the underlying diophantine equations, we conclude thus far that

$$
\int_{0}^{1}|f(\alpha) g(\alpha) K(\alpha)|^{2} d \alpha \ll P I_{1}+I_{2},
$$

where

$$
I_{1}=\int_{0}^{1}|g(\alpha) K(\alpha)|^{2} d \alpha \quad \text { and } \quad I_{2}=\int_{0}^{1}\left|F(\alpha) g(4 \alpha)^{2} K(4 \alpha)^{2}\right| d \alpha .
$$

The mean value $I_{1}$ is easily estimated. By orthogonality, one finds that $I_{1}$ is bounded above by the number of integral solutions of the equation

$$
y_{1}^{3}-y_{2}^{3}=n_{1}-n_{2},
$$

with $Q<y_{j} \leqslant 2 Q(j=1,2)$ and $n_{l} \in \mathcal{Z}(l=1,2)$. If such a solution were to exist with $y_{1} \neq y_{2}$, then one would have

$$
3 Q^{2}<\left|y_{1}^{3}-y_{2}^{3}\right|=\left|n_{1}-n_{2}\right| \leqslant Z
$$

Since the latter condition contradicts our hypothesis that $Z \leqslant Q^{2}$, we conclude that, necessarily, one has $y_{1}=y_{2}$ and $n_{1}=n_{2}$, whence

$$
I_{1} \ll Q \widehat{Z} .
$$

We estimate $I_{2}$ by means of the Hardy-Littlewood method. Define the set of major arcs $\mathfrak{M}$ to be the union of the intervals

$$
\mathfrak{M}(q, a)=\left\{\alpha \in[0,1):|q \alpha-a| \leqslant P Q^{-3}\right\},
$$

with $0 \leqslant a \leqslant q \leqslant P$ and $(a, q)=1$. Also, define the corresponding set of minor arcs by $\mathfrak{m}=[0,1) \backslash \mathfrak{M}$. The argument of the proof of Lemma 3.1 of Vaughan [Vau89] shows that, whenever $a \in \mathbb{Z}$ and $q \in \mathbb{N}$ satisfy $(a, q)=1$ and $|\alpha-a / q| \leqslant q^{-2}$, then one has

$$
\sum_{1 \leqslant h \leqslant 3 H}\left|\sum_{1 \leqslant z \leqslant 4 P} e\left(\alpha h\left(3 z^{2}+h^{2}\right)\right)\right|^{2} \ll P^{\varepsilon}\left(\frac{P^{2} H}{q+Q^{3}|q \alpha-a|}+P H+q+Q^{3}|q \alpha-a|\right) .
$$

By Cauchy's inequality, therefore, one deduces that, under the same hypotheses, one has

$$
F(\alpha) \ll P^{\varepsilon} H^{1 / 2}\left(\frac{P^{2} H}{q+Q^{3}|q \alpha-a|}+P H+q+Q^{3}|q \alpha-a|\right)^{1 / 2} .
$$

Suppose that $\alpha \in \mathfrak{m}$. By Dirichlet's approximation theorem, there exist $a \in \mathbb{Z}$ and $q \in \mathbb{N}$ with $0 \leqslant a \leqslant q \leqslant P^{-1} Q^{3},(a, q)=1$ and $|q \alpha-a| \leqslant P Q^{-3}$. But $\alpha \notin \mathfrak{M}$, so that necessarily one has $q>P$. We therefore conclude from (2.6) that

$$
\sup _{\alpha \in \mathfrak{m}}|F(\alpha)| \ll P^{\varepsilon} H^{1 / 2}\left(P H+P^{-1} Q^{3}+P\right)^{1 / 2} \ll P^{1 / 2+\varepsilon} H .
$$

On applying the latter bound in combination with Schwarz's inequality, and recalling the bound (2.5), we thus deduce from (2.4) that

$$
I_{2} \leqslant\left(\sup _{\alpha \in \mathfrak{m}}|F(\alpha)|\right) I_{1}+\int_{\mathfrak{M}}\left|F(\alpha) g(4 \alpha)^{2} K(4 \alpha)^{2}\right| d \alpha \ll P^{1 / 2+\varepsilon} H Q \widehat{Z}+I_{3}^{1 / 2} I_{4}^{1 / 2},
$$

where we write

$$
I_{3}=\int_{0}^{1}\left|F(\alpha)^{2} K(4 \alpha)^{2}\right| d \alpha \quad \text { and } \quad I_{4}=\int_{\mathfrak{M}}\left|g(4 \alpha)^{4} K(4 \alpha)^{2}\right| d \alpha .
$$

By orthogonality, the mean value $I_{3}$ is bounded above by the number of integral solutions of the equation

$$
h_{1}\left(3 z_{1}^{2}+h_{1}^{2}\right)-h_{2}\left(3 z_{2}^{2}+h_{2}^{2}\right)=4\left(n_{1}-n_{2}\right),
$$




\section{J. BRÜDERN AND T. D. WOOLEY}

with $1 \leqslant h_{i} \leqslant 3 H(i=1,2), 1 \leqslant z_{j} \leqslant 4 P(j=1,2)$ and $n_{l} \in \mathcal{Z}(l=1,2)$. Let $I_{5}$ denote the number of the latter solutions for which

$$
h_{1}^{3}-h_{2}^{3}=4\left(n_{1}-n_{2}\right),
$$

and let $I_{6}$ denote the corresponding number of solutions for which (2.9) fails to hold. Consider first any one of the $O\left(H^{2} \widehat{Z}^{2}\right)$ possible choices of $\mathbf{h}$ and $\mathbf{n}$ for which (2.9) does not hold. On writing $\nu$ for the fixed integer $4\left(n_{1}-n_{2}\right)+h_{2}^{3}-h_{1}^{3} \neq 0$, we see from $(2.8)$ that $3 h_{1} z_{1}^{2}-3 h_{2} z_{2}^{2}=\nu$. But then the elementary theory of binary quadratic forms (see, for example, Estermann [Est31]) shows that the number of possible choices for $z_{1}$ and $z_{2}$ is $O\left(P^{\varepsilon}\right)$. Thus we find that

$$
I_{6} \ll P^{\varepsilon} H^{2} \widehat{Z}^{2} \text {. }
$$

Consider next any one of the $O\left(\widehat{Z}^{2}\right)$ possible choices for $\mathbf{n}$ with $n_{1} \neq n_{2}$. By an elementary divisor function estimate, there are $O\left(H^{\varepsilon}\right)$ possible choices for $h_{1}-h_{2}$ and $h_{1}^{2}+h_{1} h_{2}+h_{2}^{2}$ satisfying (2.9), whence $O\left(H^{\varepsilon}\right)$ possible choices for $h_{1}$ and $h_{2}$. Given a fixed such choice of $\mathbf{n}$ and $\mathbf{h}$, Equation (2.8) becomes $h_{1} z_{1}^{2}=h_{2} z_{2}^{2}$, whence there are at most $4 P$ possible choices for $\mathbf{z}$. The number of solutions $\mathbf{h}, \mathbf{z}, \mathbf{n}$ counted by $I_{5}$ with $n_{1} \neq n_{2}$ is therefore $O\left(H^{\varepsilon} P \widehat{Z}^{2}\right)$. Plainly, the corresponding number with $n_{1}=n_{2}$, and consequently also $h_{1}=h_{2}$ and $z_{1}=z_{2}$, is $O(P H \widehat{Z})$. Then we conclude that

$$
I_{5} \ll H^{\varepsilon} P \widehat{Z}^{2}+P H \widehat{Z}
$$

whence, on recalling (2.10), we have

$$
I_{3} \ll P^{\varepsilon}\left(H^{2}+P\right) \widehat{Z}^{2}+P H \widehat{Z} .
$$

It remains to estimate $I_{4}$, and this requires some further notation. Write

$$
S(q, a)=\sum_{r=1}^{q} e\left(a r^{3} / q\right) \quad \text { and } \quad v(\beta ; L)=\int_{L}^{2 L} e\left(\beta \gamma^{3}\right) d \gamma
$$

We then define the function $g^{*}(\alpha)$ for $\alpha \in[0,1)$ by putting

$$
g^{*}(\alpha)=q^{-1} S(q, 4 a) v(4(\alpha-a / q) ; Q),
$$

when $\alpha \in \mathfrak{M}(q, a) \subseteq \mathfrak{M}$, and by setting $g^{*}(\alpha)=0$ otherwise. It follows from Theorem 4.1 of Vaughan [Vau97] that

and hence we deduce that

$$
\sup _{\alpha \in \mathfrak{M}}\left|g(4 \alpha)-g^{*}(\alpha)\right| \ll P^{1 / 2+\varepsilon}
$$

$$
I_{4} \ll \int_{\mathfrak{M}}\left|g(4 \alpha) g^{*}(\alpha) K(4 \alpha)\right|^{2} d \alpha+P^{1+\varepsilon} I_{1},
$$

where $I_{1}$ is defined as in (2.4). Write

$$
I_{7}=\int_{\mathfrak{M}}\left|g^{*}(\alpha)^{4} K(4 \alpha)^{2}\right| d \alpha .
$$

Then on applying Schwarz's inequality, and recalling the bound (2.5), we obtain

$$
I_{4} \ll I_{4}^{1 / 2} I_{7}^{1 / 2}+P^{1+\varepsilon} Q \widehat{Z},
$$

whence

$$
I_{4} \ll I_{7}+P^{1+\varepsilon} Q \widehat{Z} .
$$

But the methods of Vaughan [Vau97, ch. 4] suffice to establish that

$$
I_{7} \leqslant K(0)^{2} \int_{\mathfrak{M}}\left|g^{*}(\alpha)\right|^{4} d \alpha \ll Q^{1+\varepsilon} \widehat{Z}^{2},
$$




\section{ADDITIVE REPRESENTATION IN SHORT INTERVALS}

and thus we conclude from (2.13) that

$$
I_{4} \ll P^{1+\varepsilon} Q \widehat{Z}+Q^{1+\varepsilon} \widehat{Z}^{2} .
$$

On recalling our hypotheses concerning $\widehat{Z}$, we find from $(2.7),(2.11)$ and (2.14) that

$$
I_{2} \ll P^{1 / 2+\varepsilon} H Q \widehat{Z}+P^{\varepsilon}\left(P+H^{2}\right)^{1 / 2} \widehat{Z}(P Q \widehat{Z})^{1 / 2}
$$

Finally, the upper bound recorded in the statement of the lemma follows on recalling (2.3) and (2.5).

\section{Sums of six cubes in short intervals}

Our objective in this section is the proof of Theorem 1.1. The skeleton of our argument here follows closely the pattern established in previous parts of our earlier series of papers jointly with Kawada, though we require some preparation in order to bring our analysis to a successful conclusion. We consider a large natural number $N$ and a positive number $\theta$ with $17 / 63<\theta \leqslant 1$, and we write $Z=N^{\theta}$. Define next $\mathcal{Z}=\mathcal{Z}(N, Z)$ to be the set of integers $n$ with $N<n \leqslant N+Z$ that cannot be written as the sum of six cubes of natural numbers. It is convenient to abbreviate $\operatorname{card}(\mathcal{Z}(N, Z))$ to $\widehat{Z}=\widehat{Z}(N, Z)$. Write $\delta=\frac{1}{2}(\theta-17 / 63)$. We claim that the conclusion of Theorem 1.1 follows on demonstrating that, whenever $Z \leqslant N^{1 / 3}$, then one has $\widehat{Z}=O\left(Z N^{-\delta / 2}\right)$. For if $Z>N^{1 / 3}$, then we may subdivide the interval $(N, N+Z]$ into at most $\left[Z N^{-1 / 3}\right]+1$ subintervals $\left(N_{0}, N_{0}+N_{0}^{1 / 3}\right]$ of length $N_{0}^{1 / 3}$, on each of which we may infer that $\widehat{Z}\left(N_{0}, N_{0}^{1 / 3}\right) \ll N_{0}^{1 / 3-\delta / 2}$. But then we have

$$
\widehat{Z}(N, Z) \ll\left(Z N^{-1 / 3}+1\right) N^{1 / 3-\delta / 2} \ll Z N^{-\delta / 2},
$$

and this establishes our earlier claim. The conclusion of Theorem 1.1 follows on noting that

$$
\widehat{Z}(N, Z)=E_{6}(N+Z)-E_{6}(N) \text {. }
$$

Henceforth, therefore, we may suppose that $\theta \leqslant 1 / 3$.

We take $P=\frac{1}{2} N^{1 / 3}, M=P^{1 / 6}$, and define $Q$ and $H$ as in $\S 2$. It follows that

$$
Z>N^{17 / 63}>N^{1 / 6+2 \delta}>M^{3} N^{\delta}
$$

and thus there is no loss of generality in supposing that $\widehat{Z} \geqslant M^{3}$, for otherwise one has $\widehat{Z}<M^{3}<$ $Z N^{-\delta}$, and this suffices to establish Theorem 1.1 as before. In combination with the discussion of the previous paragraph, we may suppose henceforth that the hypotheses of Lemma 2.1 are satisfied.

In order to make use of recent technology employed in Waring's problem for cubes, we recall some generating functions introduced in Brüdern, Kawada and Wooley [BKW01a]. Let $\eta$ be a sufficiently small positive number depending at most on $\varepsilon$, and consider a real number $R$ with $P^{\eta / 2}<R \leqslant P^{\eta}$. We write

$$
S=P^{6 / 7}, \quad Y=P^{1 / 7},
$$

and define the generating functions

$$
\mathfrak{f}_{p}(\alpha)=\sum_{\substack{P<x \leqslant 2 P \\ p \nmid x}} e\left(\alpha x^{3}\right), \quad \mathfrak{g}(\alpha)=\sum_{S<y \leqslant 2 S} e\left(\alpha y^{3}\right), \quad \mathfrak{h}(\alpha)=\sum_{z \in \mathcal{A}(S, R)} e\left(\alpha z^{3}\right),
$$

where

$$
\mathcal{A}(S, R)=\{z \in[1, S] \cap \mathbb{Z}: p \mid z \text { and } p \text { prime } \Rightarrow p \leqslant R\}
$$




\section{J. BRÜDERN AND T. D. WOOLEY}

Finally, we define the generating function

$$
\mathcal{F}(\alpha)=\sum_{\substack{Y<p \leqslant 2 Y \\ p \equiv 2(\bmod 3)}} \mathfrak{f}_{p}(\alpha) \mathfrak{g}\left(\alpha p^{3}\right) \mathfrak{h}\left(\alpha p^{3}\right)^{2},
$$

where the summation is over prime numbers.

We may now begin our proof of Theorem 1.1 in earnest. Recall the definition of the exponential sums $f(\alpha)$ and $g(\alpha)$ from $\S 2$, and write

$$
R(n)=\int_{0}^{1} \mathcal{F}(\alpha) f(\alpha) g(\alpha) e(-n \alpha) d \alpha .
$$

Then it is apparent that, whenever $n \in \mathcal{Z}$, one has $R(n)=0$. Defining the exponential sum $K(\alpha)$ as in $\S 2$, we therefore conclude from (3.1) that

$$
\int_{0}^{1} \mathcal{F}(\alpha) f(\alpha) g(\alpha) K(-\alpha) d \alpha=\sum_{n \in \mathcal{Z}} R(n)=0 .
$$

We interpret (3.2) by means of the Hardy-Littlewood method. Write $L=(\log P)^{1 / 100}$, and define $\mathfrak{P}$ to be the union of the intervals

$$
\mathfrak{P}(q, a)=\left\{\alpha \in[0,1):|q \alpha-a| \leqslant L P^{-3}\right\},
$$

with $0 \leqslant a \leqslant q \leqslant L$ and $(a, q)=1$. We then denote the corresponding set of minor arcs by $\mathfrak{p}=[0,1) \backslash \mathfrak{P}$. We have the following lower bound for the contribution of the major arcs $\mathfrak{P}$ to the integral $R(n)$.

Lemma 3.1. Suppose that $N<n \leqslant N+Z$. Then one has

$$
\int_{\mathfrak{P}} \mathcal{F}(\alpha) f(\alpha) g(\alpha) e(-n \alpha) d \alpha \gg M^{-1} Y S^{3}(\log Y)^{-1}
$$

Proof. By following the argument of the proof of Lemma 2.1 of [BKW01a], one finds without difficulty that, for $N<n \leqslant N+Z$, one has

$$
\int_{\mathfrak{P}} \mathcal{F}(\alpha) f(\alpha) g(\alpha) e(-n \alpha) d \alpha \gg P^{-3} \mathcal{F}(0) f(0) g(0) \gg P^{-3}\left(Y P S^{3}(\log Y)^{-1}\right) P Q .
$$

The presence of the shortened exponential sum $g(\alpha)$ in place of a longer one causes no difficulties in the implicit analysis. The conclusion of the lemma consequently follows on recalling that $Q=$ $P M^{-1}$.

Employing the lower bound provided by Lemma 3.1 together with the definition of $K(\alpha)$, we see that

$$
\int_{\mathfrak{P}} \mathcal{F}(\alpha) f(\alpha) g(\alpha) K(-\alpha) d \alpha \gg \sum_{n \in \mathcal{Z}} M^{-1} Y S^{3}(\log Y)^{-1}=\widehat{Z} M^{-1} Y S^{3}(\log Y)^{-1} .
$$

On substituting the latter bound into (3.2), we conclude thus far that

$$
\left|\int_{\mathfrak{p}} \mathcal{F}(\alpha) f(\alpha) g(\alpha) K(-\alpha) d \alpha\right| \gg \widehat{Z} M^{-1} Y S^{3}(\log Y)^{-1}
$$

We now aim to obtain an upper bound for the left-hand side of the inequality (3.3), and thereby obtain an upper bound for $\widehat{Z}$.

Our next step requires a further Hardy-Littlewood dissection and further notation. We define the set of major arcs $\mathfrak{W}$ to be the union of the intervals

$$
\mathfrak{W}(q, a)=\left\{\alpha \in[0,1):|q \alpha-a| \leqslant P^{-9 / 4}\right\},
$$




\section{ADDITIVE REPRESENTATION IN SHORT INTERVALS}

with $0 \leqslant a \leqslant q \leqslant P^{3 / 4}$ and $(a, q)=1$. We then put $\mathfrak{w}=[0,1) \backslash \mathfrak{W}$. Recall the notation introduced in $(2.12)$, and define also

$$
S(q, a, p)=S(q, a)-p^{-1} S\left(q, a p^{3}\right) .
$$

Further, define the functions $\mathfrak{f}_{p}^{*}(\alpha)$ and $\mathfrak{g}_{p}^{*}(\alpha)$ for $\alpha \in[0,1)$ by putting

$$
\mathfrak{f}_{p}^{*}(\alpha)=q^{-1} S(q, a, p) v(\alpha-a / q ; P)
$$

and

$$
\mathfrak{g}_{p}^{*}(\alpha)=q^{-1} S\left(q, a p^{3}\right) v\left(p^{3}(\alpha-a / q) ; S\right),
$$

when $\alpha \in \mathfrak{W}(q, a) \subseteq \mathfrak{W}$, and by setting $\mathfrak{f}_{p}^{*}(\alpha)=0$ and $\mathfrak{g}_{p}^{*}(\alpha)=0$ otherwise. Finally, we write

$$
\mathcal{F}_{1}(\alpha)=\sum_{\substack{Y<p \leqslant 2 Y \\ p \equiv 2(\bmod 3)}} \mathfrak{f}_{p}^{*}(\alpha) \mathfrak{g}_{p}^{*}(\alpha) \mathfrak{h}\left(\alpha p^{3}\right)^{2} .
$$

The argument of [BKW01a] leading to the upper bound (3.13) of that paper reveals that

$$
\int_{\mathfrak{p} \cap \mathfrak{W}}\left|\mathcal{F}_{1}(\alpha) f(\alpha) g(\alpha)\right| d \alpha \ll M^{-1} Y S^{3} L^{-1 / 4}(\log Y)^{-1}
$$

On substituting this estimate into (3.3), we find that

$$
\widehat{Z} M^{-1} Y S^{3}(\log Y)^{-1} \ll \int_{\mathfrak{p} \cap \mathfrak{W}}\left|\left(\mathcal{F}(\alpha)-\mathcal{F}_{1}(\alpha)\right) f(\alpha) g(\alpha) K(\alpha)\right| d \alpha+\int_{\mathfrak{w}}|\mathcal{F}(\alpha) f(\alpha) g(\alpha) K(\alpha)| d \alpha .
$$

An application of Schwarz's inequality consequently yields the bound

$$
\widehat{Z} M^{-1} Y S^{3}(\log Y)^{-1} \ll\left(J_{1}^{1 / 2}+J_{2}^{1 / 2}\right) J_{3}^{1 / 2},
$$

where

$$
J_{1}=\int_{\mathfrak{W}}\left|\mathcal{F}(\alpha)-\mathcal{F}_{1}(\alpha)\right|^{2} d \alpha, \quad J_{2}=\int_{\mathfrak{w}}|\mathcal{F}(\alpha)|^{2} d \alpha, \quad J_{3}=\int_{0}^{1}|f(\alpha) g(\alpha) K(\alpha)|^{2} d \alpha .
$$

But Lemma 3.2 of [BKW01a] demonstrates that

$$
J_{1} \ll Y^{2} S^{6} P^{-19 / 14} \text { and } J_{2} \ll Y^{2} S^{6} P^{-19 / 14} \text {. }
$$

Then on applying Lemma 2.1 in order to estimate $J_{3}$, and noting that our choice of parameters ensures that $P=H^{2}$, we deduce from (3.4) that for any positive number $\varepsilon$, one has

$$
\widehat{Z} M^{-1} Y S^{3}(\log Y)^{-1} \ll Y S^{3} P^{\varepsilon-19 / 28}\left(P Q \widehat{Z}+P Q^{1 / 2} \widehat{Z}^{3 / 2}\right)^{1 / 2} .
$$

The proof of Theorem 1.1 may now be swiftly completed. We take $\varepsilon=\delta / 4$, and recall the definitions of $P, M$ and $Q$. Then we find from (3.5) that

$$
\widehat{Z} \ll M Q^{1 / 2} P^{2 \varepsilon-5 / 28} \widehat{Z}^{1 / 2}+M Q^{1 / 4} P^{2 \varepsilon-5 / 28} \widehat{Z}^{3 / 4},
$$

whence

$$
\widehat{Z} \ll M^{2} Q P^{4 \varepsilon-5 / 14}+M^{4} Q P^{8 \varepsilon-5 / 7} \ll P^{17 / 21+\delta}+P^{11 / 14+2 \delta} \ll N^{17 / 63+\delta} .
$$

Consequently, one has $\widehat{Z} \ll N^{\theta-\delta}=Z N^{-\delta}$, and so the conclusion of Theorem 1.1 follows as before.

\section{Differencing via diminishing ranges on minor arcs}

A naive application of Lemma 2.1 in pursuit of the proof of Theorem 1.2 would dictate a choice for the parameter $Z$ lying beyond that permitted by the hypotheses of the lemma. In such circumstances, a suitable analogue of Lemma 2.1 relies on a differencing process restricted to minor arcs, and it is the object of this section to establish such a mean value estimate. Before proceeding further, we 


\section{J. BRÜDERN AND T. D. WOOLEY}

require some notation, and here we economise by recycling that employed in $\S 2$. Let $P$ be a large positive number, take $M=P^{5 / 28}$, and put $Q=P M^{-1}$ and $H=P M^{-3}$. When $1 \leqslant X \leqslant P$, we define the set of major arcs $\mathfrak{N}(X)$ to be the union of the intervals

$$
\mathfrak{N}(q, a ; X)=\left\{\alpha \in[0,1):|q \alpha-a| \leqslant X Q^{-3}\right\},
$$

with $0 \leqslant a \leqslant q \leqslant X$ and $(a, q)=1$. We then put

$$
\mathfrak{M}=\mathfrak{N}(\sqrt{P}) \quad \text { and } \quad \mathfrak{m}=[0,1) \backslash \mathfrak{M} .
$$

Finally, we recall the definitions of the exponential sums $f(\alpha), g(\alpha)$ and $K(\alpha)$ from the preamble to Lemma 2.1.

Proposition 4.1. Suppose that the parameters $P, H, Z$, and the quantity $\widehat{Z}$, satisfy

$$
H^{2} \leqslant \widehat{Z} \leqslant Z \leqslant P^{3 / 2} \text {. }
$$

Then for each positive number $\varepsilon$, one has

$$
\int_{\mathfrak{m}}|f(\alpha) g(\alpha) K(\alpha)|^{2} d \alpha \ll P^{\varepsilon}\left(P Q \widehat{Z}+P H \widehat{Z} Z^{1 / 4}+P^{-5 / 4} Q^{2} \widehat{Z} Z\right) .
$$

We establish this proposition in several steps, and for ease of reference we summarise these steps in the shape of a sequence of lemmata. On considering the underlying diophantine equation, it follows from orthogonality that the integral

$$
\int_{0}^{1}|f(\alpha) g(\alpha) K(\alpha)|^{2} d \alpha
$$

is equal to the number of solutions of the diophantine equation (2.2) subject to the associated conditions. The argument initiating the proof of Lemma 2.1 shows, moreover, that whenever $\mathbf{x}, \mathbf{y}$, $\mathbf{n}$ is a solution of the latter equation counted by the above integral, then $\left|x_{1}-x_{2}\right|<3 H$. Here we note that $H=Q^{3} P^{-2}=P^{13 / 28}$. Write

$$
F(\alpha)=\sum_{|h| \leqslant 3 H} \sum_{\substack{P<x \leqslant 2 P \\ P<x+h \leqslant 2 P}} e\left(\alpha h\left(3 x^{2}+3 x h+h^{2}\right)\right) .
$$

Then on considering the underlying diophantine equations, we may infer from the above discussion that

$$
\int_{0}^{1}|f(\alpha) g(\alpha) K(\alpha)|^{2} d \alpha=\int_{0}^{1} F(\alpha)|g(\alpha) K(\alpha)|^{2} d \alpha .
$$

We aim now to show that the major arc contributions on the left- and right-hand sides of (4.2) are almost equal. From this one sees that the corresponding minor arc contributions are likewise almost equal, and since the minor arc contribution on the right-hand side of (4.2) may be bounded above via conventional technology, we obtain in this way the desired upper bound (4.1). Such a procedure occurs in work of Vaughan [Vau86b] concerning Waring's problem for sixth powers.

We begin by replacing $g(\alpha)$ by its major arc approximant. First we augment the notation $(2.12)$ by writing

$$
w(\beta)=\frac{1}{3} \sum_{Q^{3}<m \leqslant 8 Q^{3}} m^{-2 / 3} e(\beta m) .
$$

We now define $g^{*}(\alpha)$ for $\alpha \in[0,1)$ by putting

$$
g^{*}(\alpha)=q^{-1} S(q, a) w(\alpha-a / q),
$$

when $\alpha \in \mathfrak{N}(q, a ; P) \subseteq \mathfrak{N}(P)$, and by setting $g^{*}(\alpha)=0$ otherwise. Finally, when $\omega$ is a complex- 


\section{ADDITIVE REPRESENTATION IN SHORT INTERVALS}

valued measurable function on $[0,1)$, define

$$
\Xi(\omega)=\int_{\mathfrak{M}} \omega(\alpha)|g(\alpha) K(\alpha)|^{2} d \alpha-\int_{\mathfrak{M}} \omega(\alpha)\left|g^{*}(\alpha) K(\alpha)\right|^{2} d \alpha .
$$

Lemma 4.2. One has $\Xi\left(|f|^{2}\right) \ll P Q \widehat{Z}$.

Proof. From Theorem 4.1 of Vaughan [Vau97], one finds that

$$
\sup _{\alpha \in \mathfrak{M}}\left|g(\alpha)-g^{*}(\alpha)\right| \ll P^{1 / 4+\varepsilon},
$$

and hence

$$
\Xi\left(|f|^{2}\right) \ll P^{1 / 2+\varepsilon} T_{1}+P^{1 / 4+\varepsilon} T_{2},
$$

where

$$
T_{1}=\int_{0}^{1}|f(\alpha) K(\alpha)|^{2} d \alpha \quad \text { and } \quad T_{2}=\int_{\mathfrak{M}}\left|f(\alpha)^{2} g^{*}(\alpha) K(\alpha)^{2}\right| d \alpha .
$$

The estimate $T_{1} \ll P \widehat{Z}$ is immediate from the argument leading to (2.5). In order to bound $T_{2}$, we begin by observing that the methods of Vaughan [Vau97, ch. 4] establish that, whenever $\alpha \in \mathfrak{N}(q, a ; \sqrt{P}) \subseteq \mathfrak{M}$, then one has

$$
g^{*}(\alpha)^{3} \ll Q^{3}\left(q+Q^{3}|q \alpha-a|\right)^{-1} .
$$

On recalling our hypotheses on $\widehat{Z}$, it therefore follows from Lemma 2 of Brüdern [Bru88] that

$$
\int_{\mathfrak{M}}\left|g^{*}(\alpha)^{3} K(\alpha)^{2}\right| d \alpha \ll P^{\varepsilon}\left(P^{1 / 2} \widehat{Z}+\widehat{Z}^{2}\right) \ll P^{\varepsilon} \widehat{Z}^{2} .
$$

Observe next that the methods of Vaughan [Vau97, ch. 4] also show that, whenever $\alpha \in \mathfrak{N}(q, a ; \sqrt{P})$ $\subseteq \mathfrak{M}$, then

$$
f(\alpha) \ll P\left(q+P^{3}|q \alpha-a|\right)^{-1 / 3}+q^{1 / 2+\varepsilon}\left(1+P^{3}|\alpha-a / q|\right)^{1 / 2} .
$$

The hypotheses on $M$ ensure that, whenever $\alpha \in \mathfrak{N}(q, a ; \sqrt{P}) \subseteq \mathfrak{M}$, then

$$
q^{\varepsilon}\left(q+P^{3}|q \alpha-a|\right) \ll P^{1 / 2+\varepsilon} M^{3}=P^{29 / 28+\varepsilon} \ll P^{6 / 5-2 \varepsilon} .
$$

Thus, under the same conditions on $\alpha$, one has

$$
f(\alpha)^{3} \ll P^{3}\left(q+P^{3}|q \alpha-a|\right)^{-1},
$$

whence Lemma 2 of Brüdern [Bru88] yields

$$
\int_{\mathfrak{M}}\left|f(\alpha)^{3} K(\alpha)^{2}\right| d \alpha \ll P^{\varepsilon}\left(P^{1 / 2} \widehat{Z}+\widehat{Z}^{2}\right) \ll P^{\varepsilon} \widehat{Z}^{2} .
$$

Finally, an application of Hölder's inequality leads from (4.5), via (4.6) and (4.7), to the upper bound

$$
T_{2} \leqslant\left(\int_{\mathfrak{M}}\left|f(\alpha)^{3} K(\alpha)^{2}\right| d \alpha\right)^{2 / 3}\left(\int_{\mathfrak{M}}\left|g^{*}(\alpha)^{3} K(\alpha)^{2}\right| d \alpha\right)^{1 / 3} \ll P^{\varepsilon} \widehat{Z}^{2} .
$$

On substituting (4.8) along with our earlier bound for $T_{1}$ into (4.4), and noting that our hypotheses ensure that $Q \gg P^{3 / 4+\varepsilon}$ and $\widehat{Z} \ll P^{3 / 2}$, we conclude that

$$
\Xi\left(|f|^{2}\right) \ll P^{3 / 2+\varepsilon} \widehat{Z}+P^{1 / 4+\varepsilon} \widehat{Z}^{2} \ll P Q \widehat{Z} .
$$

This completes the proof of the lemma.

We next establish a conclusion similar to that of Lemma 4.2 in which $|f|^{2}$ is replaced by $F$.

Lemma 4.3. One has $\Xi(F) \ll P Q \widehat{Z}$. 


\section{J. BRÜDERN AND T. D. WOOLEY}

Proof. In view of (4.3), one has

$$
\Xi(F) \ll P^{1 / 2+\varepsilon} T_{3}+P^{1 / 4+\varepsilon} T_{4},
$$

where

$$
T_{3}=\int_{\mathfrak{M}}\left|F(\alpha) K(\alpha)^{2}\right| d \alpha \quad \text { and } \quad T_{4}=\int_{\mathfrak{M}}\left|F(\alpha) g^{*}(\alpha) K(\alpha)^{2}\right| d \alpha .
$$

It is convenient for future reference to define the function $\Upsilon(\alpha)$ on $[0,1)$ by taking

$$
\Upsilon(\alpha)=\left(q+Q^{3}|q \alpha-a|\right)^{-1},
$$

when $\alpha \in \mathfrak{N}\left(q, a ; H^{2}\right) \subseteq \mathfrak{N}\left(H^{2}\right)$, and by setting $\Upsilon(\alpha)=0$ otherwise. On isolating the term in $F(\alpha)$ corresponding to the diagonal contribution with $h=0$, it follows from the argument of the proof of Lemma 3.1 of Vaughan [Vau89] (just as in the derivation of (2.6) above) that

$$
F(\alpha) \ll P^{1+\varepsilon}+P^{1 / 2+\varepsilon} H+P^{1+\varepsilon} H \Upsilon(\alpha)^{1 / 2} \ll P^{1+\varepsilon}+P^{1+\varepsilon} H \Upsilon(\alpha)^{1 / 2},
$$

uniformly in $\alpha \in[0,1)$. When $\alpha \in \mathfrak{M}$, therefore, one has

$$
F(\alpha) \ll P^{5 / 4+\varepsilon} H \Upsilon(\alpha),
$$

whence we deduce from Lemma 2 of Brüdern [Bru88] that

$$
T_{3} \ll P^{5 / 4+\varepsilon} H \int_{\mathfrak{M}} \Upsilon(\alpha)|K(\alpha)|^{2} d \alpha \ll P^{5 / 4+\varepsilon} H Q^{\varepsilon-3}\left(P^{1 / 2} \widehat{Z}+\widehat{Z}^{2}\right) .
$$

We have already remarked that, when $\alpha \in \mathfrak{M}$, one has $g^{*}(\alpha) \ll Q \Upsilon(\alpha)^{1 / 3}$, and thus it follows from (4.11) that, whenever $\alpha \in \mathfrak{M}$,

$$
F(\alpha) g^{*}(\alpha) \ll P^{1+\varepsilon} H Q \Upsilon(\alpha)^{5 / 6} \ll H P^{13 / 12+\varepsilon} Q \Upsilon(\alpha) .
$$

Consequently, again by Lemma 2 of Brüdern [Bru88], we find from (4.10) that

$$
T_{4} \ll H P^{13 / 12+\varepsilon} Q \int_{\mathfrak{M}} \Upsilon(\alpha)|K(\alpha)|^{2} d \alpha \ll H P^{13 / 12+\varepsilon} Q^{\varepsilon-2}\left(P^{1 / 2} \widehat{Z}+\widehat{Z}^{2}\right) .
$$

Finally, on substituting (4.12) and (4.13) into (4.9), and recalling our hypotheses concerning $P, Q$ and $\widehat{Z}$, we obtain the upper bound

$$
\Xi(F) \ll P^{\varepsilon}\left(P^{7 / 4} H Q^{-3}+H P^{4 / 3} Q^{-2}\right) \widehat{Z}^{2} \ll P^{\varepsilon}\left(P^{-1 / 4}+P^{1 / 3} M^{-1}\right) \widehat{Z}^{2} \ll P Q \widehat{Z} .
$$

This completes the proof of the lemma.

Our next objective is the completion of the singular integrals implicitly associated with $F$ and $|f|^{2}$. The dependence on $q$ of the width of our major arcs generates several difficulties in this process. For the sake of conciseness, write $\Phi(\alpha)=F(\alpha)|K(\alpha)|^{2}$. Also, define the multiplicative function $\kappa(q)$ on prime powers $\pi^{l}(l \in \mathbb{N})$ by means of the relations

$$
\kappa\left(\pi^{3 l}\right)=\pi^{-l}, \quad \kappa\left(\pi^{3 l+1}\right)=2 \pi^{-l-1 / 2} \quad \text { and } \quad \kappa\left(\pi^{3 l+2}\right)=\pi^{-l-1} .
$$

We note for future reference that it follows from Lemmata 4.3-4.5 of Vaughan [Vau97] that, whenever $q \in \mathbb{N}$ and $a \in \mathbb{Z}$ satisfy $(a, q)=1$, then one has $q^{-1} S(q, a) \ll \kappa(q)$. Finally, when $\Theta$ is a complexvalued measurable function on $[0,1)$, we write

$$
\Omega(\Theta)=\sum_{1 \leqslant q \leqslant \sqrt{P}} \sum_{\substack{a=1 \\(a, q)=1}}^{q} q^{-2}|S(q, a)|^{2} \int_{-1 / 2}^{1 / 2}|w(\beta)|^{2} \Theta(\beta+a / q) d \beta .
$$

LEMma 4.4. One has

$$
\int_{\mathfrak{M}} F(\alpha)\left|g^{*}(\alpha) K(\alpha)\right|^{2} d \alpha=\Omega(\Phi)+O\left(H P^{3 / 4+\varepsilon} Q^{-1} \widehat{Z} Z\right) .
$$




\section{ADDITIVE REPRESENTATION IN SHORT INTERVALS}

Proof. Observe first that, whenever $1 \leqslant q \leqslant \sqrt{P}$ and

$$
\sqrt{P} /\left(q Q^{3}\right)<|\beta| \leqslant H^{2} /\left(q Q^{3}\right),
$$

then the estimate (4.11) shows that

$$
F(\beta+a / q) \ll P^{1+\varepsilon}+P^{3 / 4+\varepsilon} H \ll P^{3 / 4+\varepsilon} H .
$$

It therefore follows from the upper bound $w(\beta) \ll Q\left(1+Q^{3}|\beta|\right)^{-1}$, provided by Lemma 6.2 of Vaughan [Vau97], that the estimate

$$
\left|w(\beta)^{2} \Phi(\beta+a / q)\right| \ll H P^{3 / 4+\varepsilon} Q^{2}\left(1+Q^{3}|\beta|\right)^{-1}|K(\beta+a / q)|^{2}
$$

holds throughout the range (4.16). Our hypotheses ensure that $H^{2} P^{-1 / 2}>P^{1 / 4}$, and so, whenever $q \leqslant \sqrt{P}$ and $|\beta|>H^{2} /\left(q Q^{3}\right)$, it follows that

$$
|\beta|>H^{2} P^{-1 / 2} Q^{-3}>P^{1 / 4} Q^{-3} .
$$

Our earlier estimate for $w(\beta)$ therefore leads to the bound

$$
|w(\beta)|^{2} \ll Q^{2}\left(1+Q^{3}|\beta|\right)^{-2} \ll Q^{2} P^{-1 / 4}\left(1+Q^{3}|\beta|\right)^{-1},
$$

and so the upper bound (4.17) now follows, from a trivial estimate for $F(\alpha)$, also in the range $|\beta|>H^{2} /\left(q Q^{3}\right)$. We thus conclude from (4.15) that

$$
\int_{\mathfrak{M}} F(\alpha)\left|g^{*}(\alpha) K(\alpha)\right|^{2} d \alpha-\Omega(\Phi) \ll H P^{3 / 4+\varepsilon} Q^{2} \Lambda(\sqrt{P}),
$$

where we write

$$
\Lambda(U)=\sum_{1 \leqslant q \leqslant U} \kappa(q)^{2} \sum_{\substack{a=1 \\(a, q)=1}}^{q} \int_{-1 / 2}^{1 / 2}\left(1+Q^{3}|\beta|\right)^{-1}|K(\beta+a / q)|^{2} d \beta .
$$

From (4.19), we have

$$
\Lambda(U)=\sum_{1 \leqslant q \leqslant U} \kappa(q)^{2} \sum_{n, m \in \mathcal{Z}} c_{q}(n-m) \int_{-1 / 2}^{1 / 2} \frac{e(\beta(n-m))}{1+Q^{3}|\beta|} d \beta,
$$

where

$$
c_{q}(h)=\sum_{\substack{a=1 \\(a, q)=1}}^{q} e(a h / q)
$$

is Ramanujan's sum. The standard estimate $\left|c_{q}(h)\right| \leqslant(q, h)$ implies that

$$
\Lambda(U) \ll Q^{\varepsilon-3}\left(T_{5}+T_{6}\right),
$$

where

$$
T_{5}=\widehat{Z} \sum_{1 \leqslant q \leqslant U} q \kappa(q)^{2} \text { and } T_{6}=\sum_{1 \leqslant q \leqslant U} \kappa(q)^{2} \sum_{\substack{n, m \in \mathcal{Z} \\ n \neq m}}(q, n-m) .
$$

But whenever $n, m \in \mathcal{Z}$ satisfy $n \neq m$, one has $1 \leqslant|n-m| \leqslant Z$. Thus we find that

$$
\sum_{\substack{n, m \in \mathcal{Z} \\ n \neq m}}(q, n-m) \ll \widehat{Z} \sum_{1 \leqslant l \leqslant Z}(q, l) \ll \widehat{Z} Z q^{\varepsilon} .
$$

The relations (4.14) imply, moreover, that

$$
\sum_{1 \leqslant q \leqslant U} \kappa(q)^{2} \ll \prod_{p \leqslant U}\left(1+4 p^{-1}\right) \ll(\log U)^{4},
$$




\section{J. BRÜDERN AND T. D. WOOLEY}

and so we conclude from (4.20)-(4.22) that

$$
\Lambda(U) \ll U^{\varepsilon} Q^{\varepsilon-3}(U \widehat{Z}+\widehat{Z} Z) .
$$

In view of (4.18), therefore, we have the upper bound

$$
\int_{\mathfrak{M}} F(\alpha)\left|g^{*}(\alpha) K(\alpha)\right|^{2} d \alpha-\Omega(\Phi) \ll H P^{3 / 4+\varepsilon} Q^{-1}\left(P^{1 / 2} \widehat{Z}+\widehat{Z} Z\right) \ll H P^{3 / 4+\varepsilon} Q^{-1} \widehat{Z} Z,
$$

and this completes the proof of the lemma.

We also require an analogue of Lemma 4.4 in which $F(\alpha)$ is replaced by $|f(\alpha)|^{2}$. We now write $\Psi(\alpha)=|f(\alpha) K(\alpha)|^{2}$, and recall the notation defined in (4.15).

Lemma 4.5. One has

$$
\int_{\mathfrak{M}}\left|f(\alpha) g^{*}(\alpha) K(\alpha)\right|^{2} d \alpha=\Omega(\Psi)+O\left(H P^{3 / 4+\varepsilon} Q^{-1} \widehat{Z} Z\right) .
$$

Proof. We begin by observing that the methods of Vaughan [Vau97, chs. 4 and 6] demonstrate that, whenever $\beta \in\left[-\frac{1}{2}, \frac{1}{2}\right]$, and $a \in \mathbb{Z}, q \in \mathbb{N}$ satisfy $(a, q)=1$, then

$$
|f(\beta+a / q)|^{2} \ll q^{-2 / 3} P^{2}\left(1+P^{3}|\beta|\right)^{-2}+q^{1+\varepsilon}\left(1+P^{3}|\beta|\right) .
$$

Our earlier estimate for $w(\beta)$ therefore reveals that

$$
|f(\beta+a / q) w(\beta)|^{2} \ll \frac{P^{2} Q^{2}}{1+Q^{3}|\beta|}\left(T_{7}+T_{8}\right),
$$

where

$$
T_{7}=q^{-2 / 3}\left(1+P^{3}|\beta|\right)^{-2}\left(1+Q^{3}|\beta|\right)^{-1}
$$

and

$$
T_{8}=q^{1+\varepsilon} P^{-2}\left(1+P^{3}|\beta|\right)\left(1+Q^{3}|\beta|\right)^{-1} .
$$

On considering separately the cases in which $|\beta| \geqslant Q^{-3}$ and $|\beta|<Q^{-3}$, respectively, it is apparent that for all $\beta$ one has

$$
\left(1+P^{3}|\beta|\right)\left(1+Q^{3}|\beta|\right)^{-1} \ll P^{3} Q^{-3}=M^{3} .
$$

Consequently, whenever $q \leqslant \sqrt{P}$, one has

$$
T_{8} \ll P^{\varepsilon-3 / 2} M^{3}=P^{\varepsilon-27 / 28} \ll P^{-11 / 14},
$$

and if in addition $\beta$ satisfies $|\beta| \geqslant \sqrt{P} /\left(q Q^{3}\right)$, then

$$
T_{7} \ll q^{-2 / 3} M^{3}\left(\sqrt{P} M^{3} / q\right)^{-3} \ll(\sqrt{P})^{-2 / 3} M^{-6} \ll P^{-11 / 14} .
$$

Thus we conclude from (4.24) that, whenever $q \leqslant \sqrt{P}$ and $|\beta| \geqslant \sqrt{P} /\left(q Q^{3}\right)$, then

$$
|f(\beta+a / q) w(\beta)|^{2} \ll Q^{2} P^{17 / 14}\left(1+Q^{3}|\beta|\right)^{-1} .
$$

On recalling the definition of $\Psi(\alpha)$, it follows from (4.25) that

$$
\int_{\mathfrak{M}}\left|f(\alpha) g^{*}(\alpha) K(\alpha)\right|^{2} d \alpha-\Omega(\Psi) \ll Q^{2} P^{17 / 14} \Lambda(\sqrt{P}),
$$

where $\Lambda$ is defined as in (4.19). The estimate (4.23) consequently leads to the bound

$$
\int_{\mathfrak{M}}\left|f(\alpha) g^{*}(\alpha) K(\alpha)\right|^{2} d \alpha-\Omega(\Psi) \ll\left(Q^{2} P^{17 / 14}\right) Q^{\varepsilon-3}\left(P^{1 / 2} \widehat{Z}+\widehat{Z} Z\right) \ll Q^{\varepsilon-1} P^{17 / 14} \widehat{Z} Z
$$

The conclusion of the lemma follows on recalling that $Q=P^{23 / 28}$ and $H=P^{13 / 28}$. 


\section{ADDITIVE REPRESENTATION IN SHORT INTERVALS}

The main terms in the expansions established in Lemmata 4.4 and 4.5 are in fact equal, as we now demonstrate.

LEMMA 4.6. One has $\Omega(\Phi)=\Omega(\Psi)$.

Proof. Write

$$
J(l)=\int_{-1 / 2}^{1 / 2}|w(\beta)|^{2} e(\beta l) d \beta,
$$

and note that the definition of $w(\beta)$ ensures that $J(l)=0$ for $|l|>8 Q^{3}$. It is convenient also to write

$$
\chi(l)=e(l a / q) J(l) .
$$

Then it follows from the definition of $\Psi$ that

$$
\int_{-1 / 2}^{1 / 2}|w(\beta)|^{2} \Psi(\beta+a / q) d \beta=\sum_{P<x, y \leqslant 2 P} \sum_{m, n \in \mathcal{Z}} \chi\left(x^{3}-y^{3}+n-m\right) .
$$

Thus, just as in the argument leading to (4.2) above, the condition that $J(l)=0$ for $|l|>8 Q^{3}$ implies that the only values of $x$ and $y$ contributing to the sum in (4.26) are those with $|x-y|<3 H$. Following a change of variable, we deduce that

$$
\begin{aligned}
\int_{-1 / 2}^{1 / 2}|w(\beta)|^{2} \Psi(\beta+a / q) d \beta & =\sum_{|h| \leqslant 3 H} \sum_{\substack{P<x \leqslant 2 P \\
P<x+h \leqslant 2 P}} \sum_{m, n \in \mathcal{Z}} \chi\left(h\left(3 x^{2}+3 x h+h^{2}\right)+n-m\right) \\
& =\int_{-1 / 2}^{1 / 2}|w(\beta)|^{2} \Phi(\beta+a / q) d \beta .
\end{aligned}
$$

The conclusion of the lemma is now immediate from (4.15).

Collecting together the conclusions of Lemmata 4.4-4.6, we see that

$$
\int_{\mathfrak{M}}\left|f(\alpha) g^{*}(\alpha) K(\alpha)\right|^{2} d \alpha-\int_{\mathfrak{M}} F(\alpha)\left|g^{*}(\alpha) K(\alpha)\right|^{2} d \alpha \ll H P^{3 / 4+\varepsilon} Q^{-1} \widehat{Z} Z,
$$

so that in view of Lemmata 4.2 and 4.3, we have

$$
\int_{\mathfrak{M}}|f(\alpha) g(\alpha) K(\alpha)|^{2} d \alpha-\int_{\mathfrak{M}} F(\alpha)|g(\alpha) K(\alpha)|^{2} d \alpha \ll P Q \widehat{Z}+H P^{3 / 4+\varepsilon} Q^{-1} \widehat{Z} Z .
$$

Finally, making use now of the relation (4.2), we conclude thus far that

$$
\int_{\mathfrak{m}}|f(\alpha) g(\alpha) K(\alpha)|^{2} d \alpha-\int_{\mathfrak{m}} F(\alpha)|g(\alpha) K(\alpha)|^{2} d \alpha \ll P Q \widehat{Z}+H P^{3 / 4+\varepsilon} Q^{-1} \widehat{Z} Z .
$$

Our objective now is to estimate the second integral on the left-hand side of (4.27), and it transpires that a satisfactory bound here will deliver the sought-after conclusion of Proposition 4.1.

Lemma 4.7. One has

$$
\int_{\mathfrak{m}} F(\alpha)|g(\alpha) K(\alpha)|^{2} d \alpha \ll P^{\varepsilon}\left(P Q \widehat{Z}+P H \widehat{Z} Z^{1 / 4}+P^{-5 / 4} Q^{2} \widehat{Z} Z\right) .
$$

Proof. Write $\mathfrak{N}=\mathfrak{N}\left(H^{2}\right)$ and $\mathfrak{n}=[0,1) \backslash \mathfrak{N}$. Then it follows from (4.11) that

$$
\sup _{\alpha \in \mathfrak{n}}|F(\alpha)| \ll P^{1+\varepsilon} \text {. }
$$

The argument leading to (2.5) above, moreover, shows on this occasion that

$$
\int_{0}^{1}|g(\alpha) K(\alpha)|^{2} d \alpha \ll Q \widehat{Z}
$$




\section{J. BRÜDERN AND T. D. WOOLEY}

Thus we find that

$$
\int_{\mathfrak{n}} F(\alpha)|g(\alpha) K(\alpha)|^{2} d \alpha \ll\left(\sup _{\alpha \in \mathfrak{n}}|F(\alpha)|\right) \int_{0}^{1}|g(\alpha) K(\alpha)|^{2} d \alpha \ll P^{1+\varepsilon} Q \widehat{Z} .
$$

Observe next that (4.11) provides the upper bound

$$
F(\alpha) \ll P^{1+\varepsilon} H \Upsilon(\alpha)^{1 / 2},
$$

valid uniformly for $\alpha \in \mathfrak{N}$. We deduce from (4.30) that

$$
\int_{\mathfrak{N} \cap \mathfrak{m}} F(\alpha)|g(\alpha) K(\alpha)|^{2} d \alpha \ll P^{1+\varepsilon} H T_{9},
$$

where

$$
T_{9}=\int_{\mathfrak{N} \cap \mathfrak{m}} \Upsilon(\alpha)^{1 / 2}|g(\alpha) K(\alpha)|^{2} d \alpha .
$$

But Theorem 4.1 of Vaughan [Vau97] demonstrates that, for $\alpha \in \mathfrak{N}$,

$$
|g(\alpha)|^{1 / 2} \ll\left|g^{*}(\alpha)\right|^{1 / 2}+P^{\varepsilon} \Upsilon(\alpha)^{-1 / 4} .
$$

On substituting (4.33) into (4.32), we find that

$$
T_{9} \ll T_{10}+P^{\varepsilon} T_{11},
$$

where

$$
T_{10}=\int_{\mathfrak{N} \cap \mathfrak{m}} \Upsilon(\alpha)^{1 / 2}\left|g^{*}(\alpha) g(\alpha)^{3}\right|^{1 / 2}|K(\alpha)|^{2} d \alpha
$$

and

$$
T_{11}=\int_{\mathfrak{N} \mathfrak{m}} \Upsilon(\alpha)^{1 / 4}|g(\alpha)|^{3 / 2}|K(\alpha)|^{2} d \alpha .
$$

But an application of Hölder's inequality to (4.35) reveals that

$$
T_{10} \leqslant T_{9}^{3 / 4} T_{12}^{1 / 4}
$$

where

$$
T_{12}=\int_{\mathfrak{N} \cap \mathfrak{m}} \Upsilon(\alpha)^{1 / 2}\left|g^{*}(\alpha) K(\alpha)\right|^{2} d \alpha
$$

We thus conclude from (4.34) and (4.37) that

$$
T_{9} \ll P^{\varepsilon}\left(T_{11}+T_{12}\right) .
$$

From Lemma 2 of Brüdern [Bru88] one has

$$
\int_{\mathfrak{N}} \Upsilon(\alpha)|K(\alpha)|^{2} d \alpha \ll Q^{\varepsilon-3}\left(H^{2} \widehat{Z}+\widehat{Z}^{2}\right) \ll Q^{\varepsilon-3} \widehat{Z}^{2}
$$

By applying Hölder's inequality to (4.36), and recalling (4.28), we therefore find that

$$
T_{11} \leqslant\left(\int_{\mathfrak{N}} \Upsilon(\alpha)|K(\alpha)|^{2} d \alpha\right)^{1 / 4}\left(\int_{0}^{1}|g(\alpha) K(\alpha)|^{2} d \alpha\right)^{3 / 4} \ll\left(Q^{\varepsilon-3} \widehat{Z}^{2}\right)^{1 / 4}(Q \widehat{Z})^{3 / 4} \ll P^{\varepsilon} \widehat{Z}^{5 / 4} .
$$

Meanwhile, on noting that $\Upsilon(\alpha) \ll P^{-1 / 2}$ for $\alpha \in \mathfrak{N} \cap \mathfrak{m}$, we find from (4.38) that

$$
T_{12} \ll P^{-1 / 4} \int_{\mathfrak{N} \cap \mathfrak{m}}\left|g^{*}(\alpha) K(\alpha)\right|^{2} d \alpha .
$$

But on recalling (4.19) together with the upper bound (4.23), one has

$$
\int_{\mathfrak{N}}\left|g^{*}(\alpha) K(\alpha)\right|^{2} d \alpha \ll Q^{2} \Lambda\left(H^{2}\right) \ll Q^{\varepsilon-1}\left(H^{2} \widehat{Z}+\widehat{Z} Z\right) \ll Q^{\varepsilon-1} \widehat{Z} Z .
$$




\section{ADDITIVE REPRESENTATION IN SHORT INTERVALS}

We therefore conclude from (4.41) that

$$
T_{12} \ll P^{\varepsilon-1 / 4} Q^{-1} \widehat{Z} Z,
$$

so that by collecting (4.31), (4.39) and (4.40) together with this most recent upper bound, we infer that

$$
\int_{\mathfrak{N} \cap \mathfrak{m}} F(\alpha)|g(\alpha) K(\alpha)|^{2} d \alpha \ll P^{1+\varepsilon} H\left(\widehat{Z} Z^{1 / 4}+P^{-1 / 4} Q^{-1} \widehat{Z} Z\right) .
$$

The conclusion of the lemma follows from this bound together with (4.29).

The conclusion of Proposition 4.1 is now immediate on substituting the estimate provided by Lemma 4.7 into (4.27).

\section{Sums of five cubes in short intervals}

We are now equipped to prove Theorem 1.2. Let $N$ be a large natural number, write $P=\frac{1}{2} N^{1 / 3}$, and define $M, Q$ and $H$ as in $\S 4$. Let $\delta$ be a sufficiently small positive number, and put $Z=P^{10 / 7}$. Define $\mathcal{Z}=\mathcal{Z}(N, Z)$ to be the set of integers $n$ with $N<n \leqslant N+Z$ that cannot be written as the sum of five cubes of natural numbers. We aim to establish that $\widehat{Z} \ll P^{10 / 7-\delta}$. We may assume without loss of generality, therefore, that $\widehat{Z} \gg P^{10 / 7-\delta}$. As in the discussion at the beginning of $\S 3$, the conclusion of Theorem 1.2 follows in general from this restricted conclusion for the latter choice of $Z$. We continue to make use of the exponential sums $f(\alpha)$ and $g(\alpha)$ from $\S 4$, but now introduce the smooth Weyl sum

$$
t(\alpha)=\sum_{x \in \mathcal{A}\left(P, P^{\eta}\right)} e\left(\alpha x^{3}\right),
$$

where, as usual, we suppose that $\eta$ is a sufficiently small positive number. Write

$$
\rho(n)=\int_{0}^{1} f(\alpha)^{2} g(\alpha) t(\alpha)^{2} e(-n \alpha) d \alpha .
$$

Then whenever $n \in \mathcal{Z}$, one has $\rho(n)=0$. Defining the exponential sum $K(\alpha)$ as in $\S 2$ (and also, implicitly, as in $\S 4$ ), it follows from (5.1) that

$$
\int_{0}^{1} f(\alpha)^{2} g(\alpha) t(\alpha)^{2} K(-\alpha) d \alpha=0
$$

We begin with a major arc estimate.

Lemma 5.1. One has

$$
\int_{\mathfrak{M}} f(\alpha)^{2} g(\alpha) t(\alpha)^{2} K(-\alpha) d \alpha \gg P Q \widehat{Z}
$$

Proof. Write $L=(\log N)^{1 / 100}$, and define the narrow set of major arcs $\mathfrak{P}$ as in the preamble to Lemma 3.1. Also, when $1 \leqslant X \leqslant P^{3 / 2}$, let $\mathfrak{W}(X)$ denote the union of the intervals

$$
\mathfrak{W}(q, a ; X)=\left\{\alpha \in[0,1):|q \alpha-a| \leqslant X P^{-3}\right\},
$$

with $0 \leqslant a \leqslant q \leqslant X$ and $(a, q)=1$. We then put $\mathfrak{K}(X)=\mathfrak{W}(2 X) \backslash \mathfrak{W}(X)$.

The methods of Vaughan [Vau97, chs 4 and 6] show that, whenever $\alpha \in \mathfrak{N}(q, a ; \sqrt{P}) \subseteq \mathfrak{M}$, then

$$
g(\alpha) \ll Q\left(q+Q^{3}|q \alpha-a|\right)^{-1 / 3}+q^{1 / 2+\varepsilon} \ll Q q^{-1 / 3},
$$

and

$f(\alpha) \ll \kappa(q) P\left(1+P^{3}|\alpha-a / q|\right)^{-1}+q^{\varepsilon}\left(q+P^{3}|q \alpha-a|\right)^{1 / 2} \ll \kappa(q) P\left(1+P^{3}|\alpha-a / q|\right)^{-1}+P^{1 / 4+\varepsilon} M^{3 / 2}$. 


\section{J. BRÜDERN AND T. D. WOOLEY}

But whenever $\alpha \in \mathfrak{N}(q, a ; \sqrt{P}) \subseteq \mathfrak{M}$ and $\alpha \in \mathfrak{K}(X)$, one necessarily has

$$
q+P^{3}|q \alpha-a|>X,
$$

and thus it follows, under the same conditions on $\alpha$, that

$$
\begin{aligned}
f(\alpha)^{2} g(\alpha) & \ll P^{1 / 2+\varepsilon} M^{3}|g(\alpha)|+\kappa(q)^{2} P^{2} Q\left(1+P^{3}|\alpha-a / q|\right)^{-5 / 3}\left(q+P^{3}|q \alpha-a|\right)^{-1 / 3} \\
& \ll P^{1 / 2+\varepsilon} M^{3}|g(\alpha)|+\kappa(q)^{2} P^{2} Q X^{-1 / 3}\left(1+P^{3}|\alpha-a / q|\right)^{-5 / 3} .
\end{aligned}
$$

We therefore conclude that

$$
\int_{\mathfrak{M} \cap \mathfrak{K}(X)} f(\alpha)^{2} g(\alpha) t(\alpha)^{2} K(-\alpha) d \alpha \ll T_{13}+T_{14}
$$

where

$$
\begin{aligned}
& T_{13}=P^{1 / 2+\varepsilon} M^{3} \int_{0}^{1}\left|g(\alpha) t(\alpha)^{2} K(\alpha)\right| d \alpha, \\
& T_{14}=P^{2} Q X^{-1 / 3} \widehat{Z} \sum_{1 \leqslant q \leqslant 2 X} \kappa(q)^{2} \tau(q),
\end{aligned}
$$

and

$$
\tau(q)=\int_{-\infty}^{\infty} \sum_{\substack{a=1 \\(a, q)=1}}^{q}|t(\beta+a / q)|^{2}\left(1+P^{3}|\beta|\right)^{-5 / 3} d \beta .
$$

By Schwarz's inequality, one has

$$
\int_{0}^{1}\left|g(\alpha) t(\alpha)^{2} K(\alpha)\right| d \alpha \leqslant\left(\int_{0}^{1}|g(\alpha) K(\alpha)|^{2} d \alpha\right)^{1 / 2}\left(\int_{0}^{1}|t(\alpha)|^{4} d \alpha\right)^{1 / 2} .
$$

Here, the first integral on the right-hand side of (5.6) may be estimated via (4.28), and the second by means of Hua's lemma (see, for example, Lemma 2.5 of Vaughan [Vau97]), and hence we deduce from (5.4) that

$$
T_{13} \ll P^{1 / 2+\varepsilon} M^{3}(Q \widehat{Z})^{1 / 2}\left(P^{2+\varepsilon}\right)^{1 / 2} \ll P^{3 / 2+2 \varepsilon} Q^{1 / 2} M^{3} \widehat{Z}^{1 / 2} .
$$

The expression (5.5), on the other hand, may be estimated via the argument of the proof of Lemma 3.4 of Brüdern, Kawada and Wooley [BKW01a] (see also the proof of Lemma 3.3 of the latter paper). In this way, one obtains

$$
T_{14} \ll\left(P^{2} Q X^{-1 / 3} \widehat{Z}\right)\left(X^{\varepsilon} P^{-1}\right) \ll P Q \widehat{Z} X^{-1 / 4} .
$$

On substituting (5.7) and (5.8) into (5.3), and summing over the values $X=2^{i} L$ with $X \leqslant \sqrt{P}$ and $i \geqslant 0$, we may conclude thus far that

$$
\int_{\mathfrak{M} \backslash \mathfrak{P}} f(\alpha)^{2} g(\alpha) t(\alpha)^{2} K(-\alpha) d \alpha \ll P^{3 / 2+\varepsilon} Q^{1 / 2} M^{3} \widehat{Z}^{1 / 2}+P Q \widehat{Z} L^{-1 / 4} .
$$

The major arcs $\mathfrak{P}$ are sufficiently few and narrow that arguments nowadays considered routine (see Vaughan [Vau89, § 5]) suffice to establish that, for each integer $n$ with $N<n \leqslant N+Z$, one has

It follows that

$$
\int_{\mathfrak{P}} f(\alpha)^{2} g(\alpha) t(\alpha)^{2} e(-n \alpha) d \alpha \gg P Q
$$

$$
\int_{\mathfrak{P}} f(\alpha)^{2} g(\alpha) t(\alpha)^{2} K(-\alpha) d \alpha \gg \sum_{n \in \mathcal{Z}} P Q=P Q \widehat{Z} .
$$

On recalling (5.9) and noting our hypotheses on $H, P, Z$ and $\widehat{Z}$, a modest computation confirms the desired lower bound. 


\section{ADDITIVE REPRESENTATION IN SHORT INTERVALS}

We now return to the identity (5.2). Since $[0,1)$ is the disjoint union of $\mathfrak{M}$ and $\mathfrak{m}$, it follows from Lemma 5.1 that

$$
\int_{\mathfrak{m}} f(\alpha)^{2} g(\alpha) t(\alpha)^{2} K(-\alpha) d \alpha=-\int_{\mathfrak{M}} f(\alpha)^{2} g(\alpha) t(\alpha)^{2} K(-\alpha) d \alpha
$$

whence

$$
\int_{\mathfrak{m}}\left|f(\alpha)^{2} g(\alpha) t(\alpha)^{2} K(\alpha)\right| d \alpha \gg P Q \widehat{Z} .
$$

But by Schwarz's inequality, one has

$$
\int_{\mathfrak{m}}\left|f(\alpha)^{2} g(\alpha) t(\alpha)^{2} K(\alpha)\right| d \alpha \leqslant\left(\int_{\mathfrak{m}}|f(\alpha) g(\alpha) K(\alpha)|^{2} d \alpha\right)^{1 / 2}\left(\int_{0}^{1}\left|f(\alpha)^{2} t(\alpha)^{4}\right| d \alpha\right)^{1 / 2} .
$$

On considering the underlying diophantine equation, it follows from Theorem 1.2 of Wooley [Woo95] that

$$
\int_{0}^{1}\left|f(\alpha)^{2} t(\alpha)^{4}\right| d \alpha \ll P^{13 / 4-9 \delta},
$$

provided that we take $\delta<10^{-5}$. Then on substituting this estimate together with the conclusion of Proposition 4.1 into (5.11), we find that

$$
\int_{\mathfrak{m}}\left|f(\alpha)^{2} g(\alpha) t(\alpha)^{2} K(\alpha)\right| d \alpha \ll\left(P Q \widehat{Z}+P H \widehat{Z} Z^{1 / 4}+P^{-5 / 4} Q^{2} \widehat{Z} Z\right)^{1 / 2}\left(P^{13 / 8-4 \delta}\right) .
$$

Consequently, the lower bound (5.10) implies that

$$
P Q \widehat{Z} \ll P^{17 / 8-4 \delta} Q^{1 / 2} \widehat{Z}^{1 / 2}+P^{17 / 8-4 \delta} H^{1 / 2} \widehat{Z}^{1 / 2} Z^{1 / 8}+P^{1-4 \delta} Q(\widehat{Z} Z)^{1 / 2} .
$$

On recalling the definitions of the relevant parameters, we thus arrive first at the estimate

$$
\widehat{Z} \ll \widehat{Z}^{1 / 2}\left(P^{5 / 8-4 \delta} M^{1 / 2}+P^{5 / 8-4 \delta} M^{-1 / 2}\left(P^{10 / 7}\right)^{1 / 8}+P^{5 / 7-4 \delta}\right),
$$

and then

$$
\widehat{Z} \ll\left(P^{5 / 7-4 \delta}\right)^{2}+P^{10 / 7-8 \delta} \ll P^{10 / 7-8 \delta} .
$$

The relation $P \asymp N^{1 / 3}$ consequently leads to the upper bound

$$
\widehat{Z} \ll N^{10 / 21-\delta} \ll Z N^{-\delta},
$$

and the conclusion of Theorem 1.2 follows by summing over the blocks discussed at the start of $\S 3$.

\section{The asymptotic formula for sums of cubes}

We turn our attention in this section to the proof of Theorem 1.3. It transpires that the argument here is far less involved than that of $\S \S 2$ and 3 , and can be modelled closely on the analysis described in $\S 2$ of Brüdern, Kawada and Wooley [BKW01b].

Let $t$ be an integer with $1 \leqslant t \leqslant 3$, and write $s=4+t$. Also, let $\psi(\tau)=\psi_{s}(\tau)$ be a function of the type described in the statement of Theorem 1.3. We consider large positive numbers $M$ and $N$ with $M \leqslant N$, and define $\mathcal{Z}_{s}=\mathcal{Z}_{s}(N, M)$ to be the set of integers $n$ with $N<n \leqslant N+M$ for which the lower bound (1.6) holds. It is convenient to abbreviate $\operatorname{card}\left(\mathcal{Z}_{s}(N, M)\right) \operatorname{simply}$ to $Z_{s}=Z_{s}(N, M)$. As in the discussion of the first paragraph of $\S 3$, the conclusion of Theorem 1.3 follows on demonstrating that, whenever $M \leqslant \frac{1}{2} N^{2 / 3}$, then one has

$$
Z_{s} \ll N^{(9-s) / 6}(\log N)^{\varepsilon-(11-s) / 2} \psi_{s}(N)^{2} \quad(5 \leqslant s \leqslant 7) .
$$

For if $M>\frac{1}{2} N^{2 / 3}$, then we may subdivide the interval $(N, N+M]$ into at most $\left[2 M N^{-2 / 3}\right]+1$ subintervals $\left(N_{0}, N_{0}+\frac{1}{2} N_{0}^{2 / 3}\right]$ of length $\frac{1}{2} N_{0}^{2 / 3}$, on each of which we may apply the bound (6.1). 


\section{J. BRÜDERn AND T. D. WOOLEY}

We thus obtain the estimate

$$
Z_{s}(N, M) \ll\left(M N^{-2 / 3}+1\right) N^{(9-s) / 6}(\log N)^{\varepsilon-(11-s) / 2} \psi_{s}(N)^{2},
$$

and the conclusion of Theorem 1.3 follows on noting that

$$
Z_{s}(N, M)=\widetilde{E}_{s}\left(N+M ; \psi_{s}\right)-\widetilde{E}_{s}\left(N ; \psi_{s}\right) .
$$

We take $P=\frac{1}{2} N^{1 / 3}$, and define the exponential sums

$$
h(\alpha)=\sum_{1 \leqslant x \leqslant 3 P} e\left(\alpha x^{3}\right) \text { and } h_{1}(\alpha)=\sum_{P<y \leqslant 3 P} e\left(\alpha y^{3}\right) .
$$

In view of the discussion of the previous paragraph, we may suppose in what follows that $1 \leqslant M \leqslant$ $\frac{1}{2} N^{2 / 3}=2 P^{2}$. Consider an integer $n$ with $N<n \leqslant N+M$, and suppose that $x_{1}, \ldots, x_{s}$ are natural numbers satisfying the equation

$$
n=x_{1}^{3}+\cdots+x_{s}^{3} .
$$

It is apparent that one necessarily has

$$
\max _{1 \leqslant i \leqslant s} x_{i} \geqslant(n / s)^{1 / 3}>(N / 8)^{1 / 3}=P,
$$

as well as

$$
\max _{1 \leqslant i \leqslant s} x_{i} \leqslant n^{1 / 3} \leqslant(2 N)^{1 / 3}<3 P .
$$

By orthogonality, it follows from (6.2) that

$$
\int_{0}^{1}\left(h(\alpha)-h_{1}(\alpha)\right)^{s} e(-n \alpha) d \alpha=0,
$$

and from (6.3) we see that

$$
R_{s}(n)=\int_{0}^{1} h(\alpha)^{s} e(-n \alpha) d \alpha .
$$

On substituting the former into the latter, it follows that

$$
\begin{aligned}
R_{s}(n) & =\int_{0}^{1}\left(h(\alpha)^{s}-\left(h(\alpha)-h_{1}(\alpha)\right)^{s}\right) e(-n \alpha) d \alpha \\
& =\sum_{j=1}^{s}(-1)^{j+1}\left(\begin{array}{l}
s \\
j
\end{array}\right) \mathcal{R}_{s, j}([0,1)),
\end{aligned}
$$

where we write

$$
\mathcal{R}_{s, j}(\mathfrak{B})=\int_{\mathfrak{B}} h_{1}(\alpha)^{j} h(\alpha)^{s-j} e(-n \alpha) d \alpha .
$$

Let $\mathfrak{M}$ denote the union of the intervals

$$
\mathfrak{M}(q, a)=\left\{\alpha \in[0,1):|q \alpha-a| \leqslant P(6 N)^{-1}\right\},
$$

with $0 \leqslant a \leqslant q \leqslant P / 6$ and $(a, q)=1$. Also, recall the definition (1.5) of the singular series $\mathfrak{S}_{s}(n)$. Then it follows from the methods underlying the proof of Theorem 4.4 of Vaughan [Vau97] that there is a positive number $\nu$ such that, whenever $N<n \leqslant N+M$, one has

$$
\sum_{j=1}^{s}(-1)^{j+1}\left(\begin{array}{l}
s \\
j
\end{array}\right) \mathcal{R}_{s, j}(\mathfrak{M})=\frac{\Gamma(4 / 3)^{s}}{\Gamma(s / 3)} \mathfrak{S}_{s}(n) n^{s / 3-1}+O\left(n^{s / 3-1-\nu}\right) .
$$

Since this observation is not quite transparent, we offer some additional explanation. On following the argument of the proof of Theorem 4.4 of Vaughan [Vau97], one finds that for $1 \leqslant j \leqslant s$ one has

$$
\mathcal{R}_{s, j}(\mathfrak{M})=J_{s, j}(n) \mathfrak{S}_{s}(n)+O\left(n^{s / 3-1-\nu}\right),
$$


where

$$
J_{s, j}(n)=\int_{-\infty}^{\infty} \int_{\mathfrak{B}_{s, j}} e\left(\beta\left(\gamma_{1}^{3}+\cdots+\gamma_{s}^{3}-n\right)\right) d \gamma d \beta,
$$

and

$$
\mathfrak{B}_{s, j}=[P, 3 P]^{j} \times[0,3 P]^{s-j} .
$$

It follows that

$$
\sum_{j=1}^{s}(-1)^{j+1}\left(\begin{array}{l}
s \\
j
\end{array}\right) J_{s, j}(n)=J(3 P)-J(P),
$$

where we write

$$
J(Q)=\int_{-\infty}^{\infty} \int_{[0, Q]^{s}} e\left(\beta\left(\gamma_{1}^{3}+\cdots+\gamma_{s}^{3}-n\right)\right) d \gamma d \beta .
$$

But an application of Fourier's integral formula, just as in the classical treatment, reveals that

$$
J(3 P)=\frac{\Gamma(4 / 3)^{s}}{\Gamma(s / 3)} n^{s / 3-1}
$$

Meanwhile, on noting that $s P^{3}<n$, a second application of Fourier's integral formula leads to the conclusion that

$$
J(P)=0 .
$$

The desired formula (6.6) now follows from (6.7)-(6.10).

Now write $\mathfrak{m}=[0,1) \backslash \mathfrak{M}$. Then for $n \in \mathcal{Z}_{s}(N, M)$, on recalling our implicit hypothesis that $\psi_{s}(n)=O\left(n^{\delta}\right)$ for some sufficiently small positive number $\delta$, it follows from (1.6), (6.4) and (6.6) that

$$
\left|\sum_{j=1}^{s}(-1)^{j+1}\left(\begin{array}{l}
s \\
j
\end{array}\right) \mathcal{R}_{s, j}(\mathfrak{m})\right|>\frac{1}{2} n^{s / 3-1} \psi_{s}(n)^{-1},
$$

whence

$$
\sum_{j=1}^{s}\left(\begin{array}{l}
s \\
j
\end{array}\right)\left|\mathcal{R}_{s, j}(\mathfrak{m})\right|>\frac{1}{2} n^{s / 3-1} \psi_{s}(n)^{-1} .
$$

Define the complex numbers $\eta_{s, j}(n)$ by taking $\eta_{s, j}(n)=0$ for $n \notin \mathcal{Z}_{s}(N, M)$, and when $n \in \mathcal{Z}_{s}(N, M)$ by means of the equation

$$
\left|\int_{\mathfrak{m}} h_{1}(\alpha)^{j} h(\alpha)^{s-j} e(-n \alpha) d \alpha\right|=\eta_{s, j}(n) \int_{\mathfrak{m}} h_{1}(\alpha)^{j} h(\alpha)^{s-j} e(-n \alpha) d \alpha .
$$

Plainly, one has $\left|\eta_{s, j}(n)\right| \leqslant 1$ for every natural number $n$. In view of (6.5) and (6.11), we deduce that

$$
\begin{aligned}
N^{s / 3-1} \psi_{s}(N)^{-1} Z_{s}(N, M) & \ll \sum_{j=1}^{s}\left(\begin{array}{l}
s \\
j
\end{array}\right) \sum_{N<n \leqslant N+M} \eta_{s, j}(n) \int_{\mathfrak{m}} h_{1}(\alpha)^{j} h(\alpha)^{s-j} e(-n \alpha) d \alpha \\
& =\sum_{j=1}^{s}\left(\begin{array}{l}
s \\
j
\end{array}\right) \int_{\mathfrak{m}} h_{1}(\alpha)^{j} h(\alpha)^{s-j} K_{s, j}(-\alpha) d \alpha,
\end{aligned}
$$

where

$$
K_{s, j}(\alpha)=\sum_{N<n \leqslant N+M} \eta_{s, j}(n) e(n \alpha) .
$$

Thus we conclude that

$$
N^{s / 3-1} \psi_{s}(N)^{-1} Z_{s}(N, M) \ll \max _{1 \leqslant j \leqslant s} \int_{\mathfrak{m}}\left|h_{1}(\alpha)^{j} h(\alpha)^{s-j} K_{s, j}(\alpha)\right| d \alpha .
$$




\section{J. BRÜDERN AND T. D. WOOLEY}

Let $J$ be the index for which the maximum is achieved on the right-hand side of (6.13). Then by applying Hölder's inequality, one obtains

$$
\int_{\mathfrak{m}}\left|h_{1}(\alpha)^{J} h(\alpha)^{s-J} K_{s, J}(\alpha)\right| d \alpha \leqslant \mathcal{I}_{1}^{1 / 2} \mathcal{I}_{2}^{(J-1) /(2 s-2)} \mathcal{I}_{3}^{(s-J) /(2 s-2)},
$$

where

$$
\mathcal{I}_{1}=\int_{0}^{1}\left|h_{1}(\alpha) K_{s, J}(\alpha)\right|^{2} d \alpha, \quad \mathcal{I}_{2}=\int_{\mathfrak{m}}\left|h_{1}(\alpha)\right|^{2 s-2} d \alpha, \quad \mathcal{I}_{3}=\int_{\mathfrak{m}}|h(\alpha)|^{2 s-2} d \alpha .
$$

But on recalling that $s=4+t$, one finds that

$$
\mathcal{I}_{2} \leqslant\left(\sup _{\alpha \in \mathfrak{m}}\left|h_{1}(\alpha)\right|\right)^{2 t-2} \int_{\mathfrak{m}}\left|h_{1}(\alpha)\right|^{8} d \alpha .
$$

The methods of Vaughan [Vau86a], as refined by Boklan [Bok93], yield the upper bound

$$
\int_{\mathfrak{m}}\left|h_{1}(\alpha)\right|^{8} d \alpha \ll P^{5}(\log P)^{\varepsilon-3},
$$

for any positive number $\varepsilon$. Meanwhile, on combining the refined estimates of Hall and Tenenbaum [HT88] for Hooley's $\Delta$-function with the proof of Lemma 1 of Vaughan [Vau86a], one obtains

$$
\sup _{\alpha \in \mathfrak{m}}\left|h_{1}(\alpha)\right| \ll P^{3 / 4}(\log P)^{1 / 4+\varepsilon} .
$$

Thus we conclude from (6.16) that

$$
\begin{aligned}
\mathcal{I}_{2} & \ll\left(P^{3 / 4}(\log P)^{1 / 4+\varepsilon}\right)^{2 t-2} P^{5}(\log P)^{\varepsilon-3} \\
& \ll P^{(3 s-5) / 2}(\log P)^{\varepsilon+(s-11) / 2},
\end{aligned}
$$

and a similar argument shows likewise that

$$
\mathcal{I}_{3} \ll P^{(3 s-5) / 2}(\log P)^{\varepsilon+(s-11) / 2} .
$$

In order to estimate the mean value (6.15), we begin by noting, from orthogonality, that it follows from (6.12) that $\mathcal{I}_{1}$ is bounded above by the number of integral solutions of the equation

$$
y_{1}^{3}-y_{2}^{3}=n_{1}-n_{2},
$$

with $P<y_{i} \leqslant 3 P(i=1,2)$ and $n_{l} \in \mathcal{Z}_{s}(N, M)(l=1,2)$. But whenever $y_{1} \neq y_{2}$, it follows from the latter conditions that

$$
\left|y_{1}^{3}-y_{2}^{3}\right|>3 P^{2}>M \geqslant\left|n_{1}-n_{2}\right| .
$$

We are forced to conclude that the only solutions of Equation (6.19) satisfy $y_{1}=y_{2}$ and $n_{1}=n_{2}$, whence

$$
\mathcal{I}_{1} \ll P Z_{s}(N, M) .
$$

On substituting $(6.17),(6.18)$ and (6.20) into (6.14), we obtain the estimate

$$
\max _{1 \leqslant j \leqslant s} \int_{\mathfrak{m}}\left|h_{1}(\alpha)^{j} h(\alpha)^{s-j} K_{s, j}(\alpha)\right| d \alpha \ll\left(P Z_{s}\right)^{1 / 2}\left(P^{(3 s-5) / 2}(\log P)^{\varepsilon+(s-11) / 2}\right)^{1 / 2},
$$

whence by (6.13), we have

$$
N^{(s-3) / 3} \psi_{s}(N)^{-1} Z_{s} \ll P^{(3 s-3) / 4}(\log P)^{\varepsilon+(s-11) / 4} Z_{s}^{1 / 2} .
$$

On recalling that $P=\frac{1}{2} N^{1 / 3}$, we therefore conclude that

$$
Z_{s} \ll P^{(9-s) / 2}(\log P)^{\varepsilon-(11-s) / 2} \psi_{s}(N)^{2},
$$

and the desired conclusion (6.1) follows immediately. This completes the proof of Theorem 1.3. 


\section{ADDITIVE REPRESENTATION IN SHORT INTERVALS}

\section{ACKNowledgements}

The referees of this paper made numerous careful suggestions, and the authors gratefully acknowledge the improvements resulting from the revision of the first version of this manuscript.

\section{REFERENCES}

BHP97 R. C. Baker, G. Harman and J. Pintz, The exceptional set for Goldbach's problem in short intervals, in Sieve methods, exponential sums, and their applications in number theory (Cardiff, 1995), eds G. Greaves, G. Harman and M. Huxley (Cambridge University Press, Cambridge, 1997), 1-54.

Bok93 K. D. Boklan, A reduction technique in Waring's problem, I, Acta Arith. 65 (1993), 147-161.

Bru88 J. Brüdern, A problem in additive number theory, Math. Proc. Cambridge Philos. Soc. 103 (1988), $27-33$.

Bru91 J. Brüdern, On Waring's problem for cubes, Math. Proc. Cambridge Philos. Soc. 109 (1991), 229-256.

BKW00 J. Brüdern, K. Kawada and T. D. Wooley, Additive representation in thin sequences, II: the binary Goldbach problem, Mathematika 47 (2000), 117-125 (2002).

BKW01a J. Brüdern, K. Kawada and T. D. Wooley, Additive representation in thin sequences, I: Waring's problem for cubes, Ann. Sci. École Norm. Sup. (4) 34 (2001), 471-501.

BKW01b J. Brüdern, K. Kawada and T. D. Wooley, Additive representation in thin sequences, III: asymptotic formulae, Acta Arith. 100 (2001), 267-289.

BKW02 J. Brüdern, K. Kawada and T. D. Wooley, Additive representation in thin sequences, VI: representing primes, and related problems, Glasg. Math. J. 44 (2002), 419-434.

BKW03 J. Brüdern, K. Kawada and T. D. Wooley, Additive representation in thin sequences, V: mixed problems of Waring's type, Math. Scand. 92 (2003), 181-209.

BW95 J. Brüdern and N. Watt, On Waring's problem for four cubes, Duke Math. J. 77 (1995), 583-606.

Dav39 H. Davenport, On Waring's problem for cubes, Acta Math. 71 (1939), 123-143.

Est31 T. Estermann, Einige Sätze über quadratfreie Zahlen, Math. Ann. 105 (1931), 653-662.

HT88 R. Hall and G. Tenenbaum, Divisors (Cambridge University Press, Cambridge, 1988).

Kaw96 K. Kawada, On the sum of four cubes, Mathematika 43 (1996), 323-348.

LP96 A. Languasco and A. Perelli, A pair correlation hypothesis and the exceptional set in Goldbach's problem, Mathematika 43 (1996), 349-361.

Lin43 J. V. Linnik, On the representation of large numbers as sums of seven cubes, Mat. Sbornik 12 (1943), 218-224.

PP93 A. Perelli and J. Pintz, On the exceptional set for Goldbach's problem in short intervals, J. London Math. Soc. (2) 47 (1993), 41-49.

Ram73 K. Ramachandra, On the number of Goldbach numbers in small intervals, J. Indian Math. Soc. 37 (1973), 157-170.

Vau86a R. C. Vaughan, On Waring's problem for cubes, J. reine angew. Math. 365 (1986), 122-170.

Vau86b R. C. Vaughan, On Waring's problem for sixth powers, J. London Math. Soc. (2) 33 (1986), 227-236.

Vau89 R. C. Vaughan, A new iterative method in Waring's problem, Acta Math. 162 (1989), 1-71.

Vau97 R. C. Vaughan, The Hardy-Littlewood method, second edition (Cambridge University Press, Cambridge, 1997).

Woo95 T. D. Wooley, Breaking classical convexity in Waring's problem: sums of cubes and quasi-diagonal behaviour, Invent. Math. 122 (1995), 421-451.

Woo00 T. D. Wooley, Sums of three cubes, Mathematika 47 (2000), 53-61.

Woo02 T. D. Wooley, Slim exceptional sets for sums of cubes, Canad. J. Math. 54 (2002), 417-448. 


\section{ADDITIVE REPRESENTATION IN SHORT INTERVALS}

J. Brüdern Joerg.Bruedern@mathematik.uni-stuttgart.de

Mathematisches Institut A, Universität Stuttgart, Postfach 8011 40, D-70511 Stuttgart, Germany

T. D. Wooley wooley@math.Isa.umich.edu

Department of Mathematics, University of Michigan, East Hall, 525 East University Avenue, Ann Arbor, MI 48109-1109, USA 